\title{
Screening of Brinjal Germplasm against Wilt Complex Disease
}

\author{
Richa Sharma $^{1 *}$, V.K. Razdan ${ }^{1}$, Stanzin Dorjey ${ }^{2}$, Prerna Gupta ${ }^{3}$ and Seethiya Mahajan ${ }^{1}$ \\ ${ }^{1}$ Division of Plant Pathology, Sher-e-Kashmir University of Agricultural Sciences and \\ Technology of Jammu, Chatha, Jammu-180009, India \\ ${ }^{2}$ Division of Plant Pathology, Sher-e-Kashmir University of Agricultural Sciences and \\ Technology of Kashmir, SKUAST-Kashmir, India \\ ${ }^{3}$ Division of Food Technology and Nutrition, School of Agriculture, Lovely Professional \\ University, India \\ *Corresponding author
}

\section{A B S T R A C T}

\begin{tabular}{|l|}
\hline Key w o r d s \\
Brinjal, Germplasm, \\
Screening, Soil \\
borne pathogens, \\
Wilt complex
\end{tabular}

\section{Introduction}

Brinjal or eggplant (Solanum melongena $\mathrm{L}$.) is a member of solanaceae family and a common vegetable crop grown all over the world. India is considered to be the centre of its origin (Zeven and Zhukovsky, 1975) and many varieties of brinjal, varying in shape, size and colour of the fruit are grown here. India is the second largest producer of brinjal in the world next to China (Anonymous, 2009). The global area under brinjal cultivation during the year 2015-16 has been estimated at 663 million hectare with production of about 12515 million tonnes (Anonymous, 2016). Brinjal cultivation in India is estimated to cover about 8.14 per cent vegetable area with a contribution of nine per cent to total vegetable production. Jammu and Kashmir accounts for an area of 2.0 thousand hectare with a production of 45.24 thousand tones.

Cultivation of resistant genotypes is the best method of managing the disease. Besides resistance to soil borne pathogens, these lines could be used for hybridization programme to 
evolve cultivars possessing desirable traits As brinjal is susceptible to several diseases such as damping off, wilt, root rot, collar rot, etc. the development of resistant varieties is a major challenge of which Fusarium oxysporum f. sp. melonogena, Rhizoctonia solani, Sclerotium rolfsii, are the most common. So, use of synthetic chemicals probably can kill the beneficial soil microflora.

Therefore, resistant varieties of crop plants will result to escape such diseases. The present investigations were undertaken to screen out the available germplasm of brinjal against wilt complex diseases in field and pot conditions.

\section{Materials and Methods}

The experiment was laid in Randomized Block Design with three replications, during 2014 and 2015, to evaluate thirty lines under field conditions. Another set of experiment with the select germplasm was laid in pots under glasshouse conditions.

Healthy brinjal seeds of susceptible brinjal cultivar Pusa Purple Long (PPL) were sown in $2 \times 3$ meter plots for raising nursery. Onemonth-old seedlings were transplanted to main field in plots with the spacing of $60 \times 45 \mathrm{~cm}$, in four rows of four plants each. Each plot was separated by a row of susceptible brinjal variety PPL, to ensure the availability of adequate inoculum for the development of infection.

To evaluate the germplasm under glasshouse conditions, Fusarium oxysporum f. sp. melongenae, Rhizoctonia solani and Sclerotium rolfsii, mass multiplied on sterilize sorghum grains, were added to the pots, each @ $5 \mathrm{~g} / \mathrm{kg}$ of soil. Potted soil were mixed with FYM in 2:1 ratio and sterilized with 5 per cent formalin by thoroughly mixing and then covering it with a polyethylene sheet for 72 hours. Later it was spread for 48 hours to release formalin fumes. One brinjal plant per pot was maintained. All the agronomic practices were followed as per the SKUASTJammu package of practices.

The data was recorded at 30, 45 and 60 days after inoculation (DAI) and per cent disease incidence was calculated as mentioned earlier (3.1). The reaction of each genotype was categorized on the basis of per cent disease incidence. The disease reaction was calculated as per the scale suggested by Mew and Ho (1976).

\section{Disease reaction: Wilt incidence}

Resistant (R): <20\% wilting

Moderately resistant (MR): 20 to $40 \%$ wilting

Moderately Susceptible (MS): 41 to $60 \%$ wilting

Susceptible: $>60 \%$ wilting

\section{Results and Discussion}

Germplasm obtained from different sources were evaluated against the soil borne pathogens of brinjal viz., $F$. oxysporum f. sp. melongenae, $R$. solani and $S$. rofsii (during the year 2014 and 2015) (Table 1). The pooled data prsented in the Table reveal that under field conditions, the minimum $(7.78 \%)$ wilt complex was recorded in Arka Nidhi. It was followed by Arka Neelkanth, Pusa Purple Cluster, Swaran Shamli, Banaras Giantand Pusa Purple Round, in which wilt complex recorded was 9.25, 9.60, 12.50, 12.50and 15.00 per cent, respectively. However, the germplasm, Pusa Hybrid-13, Shamli, Unnati, Pusa Hybrid-5, Nisha Improved, Punjab Sadabahar, Navkiran, Pusa Hybrid-6, Brinjal Round and Pant Rituraj recorded wilt complex of 44.20, 45.60, 45.80, 46.00, 51.00, 52.00, 
$53.00,54.20,54.20$ and 55.20 per cent, respectively. The germplasm viz., Black Beauty, Kokila, Daksh, Azad Kranti, Pant Samrat, Punjab Barsati, Krishna, Mahadeva, Manisha, Utsav and Pusa Purple Long resulted in $60.50,60.50,60.50,60.80,61.70,62.50$, $62.50,62.70,64.60,66.50$ and 68.45 per cent wilt complex, respectively.

Regarding the disease reaction on different germplasms, the Table 1 further reveal that under field conditions, the germplasm that could be categorised as resistant were Pusa Purple Round, Pusa Purple Cluster, Swaran Shamli, Arka Nidhi, Banaras Giant and Arka Neelkanth, where the wilt incidence ranged from 0.00 to 20.00 per cent.

However, Pusa Kranti, Green Long and Brinjal Hybrid Gole exhibited wilt incidence ranging from 21.00 to 40.00 per cent, therefore were categorised as moderately resistant. Nisha Improved, Pusa Hybrid-6, Shamli, Punjab Sadabahar, Brinjal Round, Pusa Hybrid-13 and Unnati were categorized as moderately susceptible, as they recorded wilt incidence ranging from 40.00 to 60.00 per cent.

Pusa Purple Long, Navkiran, Pant Samrat, Kokila, Pant Rituraj, Manisha, Daksh, Utsav, Mahadeva, Punjab Barsati, Black Beauty, Krishna and Azad Kranti were categorized as susceptible as they exhibited the wilt incidence of more than 60 per cent. The consolidated categorization of the germplasm as per the disease reaction has been presented in Table 16.

During the year 2014 and 2015, the pot experiment was also conducted to screen the obtained germplasm lines of brinjal and the pooled data presented in Table 1 reveal that minimum wilt complex was recorded in case of germplasm Arka Nidhi (13.00\%). The germplasms viz., Pusa Purple Cluster, Arka
Neelkanth, Swaran Shamli and Banaras Giant recorded 13.60, 16.20, 17.75 and 17.75 per cent, wilt complex, respectively. Whereas, the germplasms Pusa Purple Round and Pusa Kranti recorded 27.80 and 39.00 per cent, wilt complex, respectively.

However, the germplasm viz., Green long, Brinjal Hybrid Gole, Pusa Hybrid-5, Pusa Hybrid-13, Unnati, Nisha Improved, Shamli, Pusa Hybrid-6 and Punjab Sadabahar recorded wilt complex of 43.80, 49.60, 52.00, 52.50, 54.17, 55.80, 56.90, 57.50 and 57.20 per cent, respectively.

The germplasm viz., Pant Rituraj, Brinjal Round, Navkiran, Mahadeva, Pant Samrat, Krishna, Punjab Barsati, Kokila, Azad Kranti, Black Beauty, Daksh, Manisha, Utsav and Pusa Purple Long exhibited 60.30, 60.80, $64.60,64.70,65.00,65.80,65.83,66.33$, $67.00,67.33,67.50,68.25,72.50,73.50$ and 77.50 per cent wilt complex, respectively.

Under pot conditions, Pusa Purple Cluster, Arka Nidhi, Swaran Shamli, Banaras Giant andArka Neelkanth were categorised asresistant,as the wilt incidence recorded in them ranged from 0.00 to 20.00 per cent.

Pusa Kranti and Pusa Purple Round were categorised as moderately resistant with wilt incidence from 20.00 to 40.00 per cent. Nisha Improved, Pusa Hybrid-5, Pusa Hybrid-6, Punjab Sadabahar, Shamli, Green long, Brinjal Hybrid Gole and Unnati recorded incidence ranging from 41.00 to 60.00 per cent, hence were categorized as moderately susceptible.

Pusa Purple Long, Navkiran, Pant Samrat, Pant Rituraj, Brinjal Round, Kokila, Manisha, Daksh, Utsav, Mahadeva, Uttara, Azad Kranti, Krishna, Punjab Barsati and Black Beauty were categorized as susceptible, as they showed wilt incidence of more than 61.00 per cent. The data is also presented in Table 2 
Int.J.Curr.Microbiol.App.Sci (2018) 7(8): 1534-1539

Table.1 Evaluation of brinjal germplasm against wilt complex under field and pot experiments

\begin{tabular}{|c|c|c|c|c|c|c|c|c|}
\hline \multirow[t]{3}{*}{ Germplasm } & \multicolumn{8}{|c|}{ Wilt Incidence (\%) } \\
\hline & \multicolumn{4}{|c|}{ Under Field conditions } & \multicolumn{4}{|c|}{ Under Pot experiment } \\
\hline & 2014 & 2015 & Pooled & $\begin{array}{c}\text { Disease } \\
\text { Reaction }\end{array}$ & 2014 & 2015 & Pooled & $\begin{array}{c}\text { Disease } \\
\text { Reaction }\end{array}$ \\
\hline Pusa Purple Long & 68.90 & 68.00 & 68.45 & S & 80.00 & 75.00 & 77.50 & $\mathrm{~S}$ \\
\hline Pusa Hybrid -5 & 48.00 & 44.00 & 46.00 & MS & 54.00 & 50.00 & 52.00 & MS \\
\hline Pusa Kranti & 30.00 & 26.00 & 28.00 & MR & 40.00 & 38.00 & 39.00 & MR \\
\hline Kokila & 60.00 & 61.00 & 60.50 & $\mathrm{~S}$ & 68.00 & 66.00 & 67.00 & $\mathrm{~S}$ \\
\hline Navkiran & 54.00 & 52.00 & 53.00 & MS & 66.00 & 63.33 & 64.70 & S \\
\hline Manisha & 66.67 & 62.50 & 64.60 & $\mathrm{~S}$ & 75.00 & 70.00 & 72.50 & S \\
\hline Daksh & 61.00 & 60.00 & 60.50 & S & 68.00 & 68.50 & 68.25 & S \\
\hline$\overline{\text { Utsav }}$ & 68.00 & 66.67 & 67.33 & S & 75.00 & 72.00 & 73.50 & S \\
\hline Mahadeva & 62.00 & 63.33 & 62.70 & S & 65.00 & 65.00 & 65.00 & S \\
\hline Arka Nidhi & 6.67 & 8.89 & 7.78 & $\mathrm{R}$ & 14.00 & 12.00 & 13.00 & $\mathrm{R}$ \\
\hline Azad Kranti & 61.67 & 60.00 & 60.80 & S & 66.67 & 68.00 & 67.33 & $\mathrm{~S}$ \\
\hline $\begin{array}{l}\text { Punjab } \\
\text { Sadabahar }\end{array}$ & 50.00 & 54.00 & 52.00 & MS & 56.00 & 58.33 & 57.20 & MS \\
\hline Shamli & 46.67 & 44.44 & 45.60 & MS & 58.33 & 55.55 & 56.90 & MS \\
\hline Krishna & 60.00 & 58.33 & 60.50 & $\mathrm{~S}$ & 66.67 & 65.00 & 65.83 & $\mathrm{~S}$ \\
\hline Pant Samrat & 63.33 & 60.00 & 61.70 & S & 65.00 & 66.67 & 65.80 & S \\
\hline Pant Rituraj & 56.00 & 54.44 & 55.20 & S & 62.50 & 58.00 & 60.30 & S \\
\hline Brinjal Round & 56.67 & 51.67 & 54.20 & MS & 61.67 & 60.00 & 60.80 & $\mathrm{~S}$ \\
\hline Nisha Improved & 50.00 & 52.00 & 51.00 & MS & 55.00 & 56.67 & 55.80 & MS \\
\hline Pusa Hybrid- 6 & 51.67 & 56.67 & 54.20 & MS & 56.67 & 58.33 & 57.50 & MS \\
\hline $\begin{array}{l}\text { Pusa Purple } \\
\text { cluster }\end{array}$ & 9.00 & 10.12 & 9.60 & $\mathrm{R}$ & 12.12 & 15.00 & 13.60 & $\mathrm{R}$ \\
\hline Swaran Shamli & 11.67 & 13.33 & 12.50 & $\mathrm{R}$ & 18.00 & 17.50 & 17.75 & $\mathrm{R}$ \\
\hline $\begin{array}{l}\text { Pusa Purple } \\
\text { Round }\end{array}$ & 18.33 & 11.67 & 15.00 & $\mathrm{R}$ & 28.33 & 27.27 & 27.80 & MR \\
\hline Green Long & 31.67 & 35.00 & 33.30 & MR & 42.00 & 45.50 & 43.80 & MS \\
\hline $\begin{array}{l}\text { Brinjal Hybrid } \\
\text { Gole }\end{array}$ & 37.50 & 35.00 & 36.25 & MR & 44.17 & 55.00 & 49.60 & MS \\
\hline Pusa Hybrid-13 & 45.00 & 43.33 & 44.20 & MS & 51.67 & 53.33 & 52.50 & MS \\
\hline Unnati & 50.00 & 41.67 & 45.80 & MS & 58.33 & 50.00 & 54.17 & MS \\
\hline Punjab Barsati & 61.67 & 63.33 & 62.50 & $\mathrm{~S}$ & 66.67 & 66.00 & 66.33 & $\mathrm{~S}$ \\
\hline Banaras Giant & 11.67 & 13.33 & 12.50 & $\mathrm{R}$ & 18.00 & 17.50 & 17.75 & $\mathrm{R}$ \\
\hline Black Beauty & 60.00 & 61.00 & 60.50 & $\mathrm{~S}$ & 67.00 & 68.00 & 67.50 & $\mathrm{~S}$ \\
\hline Arka Neelkanth & 10.50 & 8.00 & 9.25 & $\mathrm{R}$ & 18.52 & 13.79 & 16.20 & $\mathrm{R}$ \\
\hline
\end{tabular}


Table.2 Reaction of brinjal germplasm against wilt complex disease under field and pot experiment

\begin{tabular}{|c|c|c|c|}
\hline Rating & $\begin{array}{l}\text { Disease } \\
\text { incidence }\end{array}$ & Field conditions & Pot experiment \\
\hline Resistant & $<20 \%$ & $\begin{array}{l}\text { Arka Nidhi, Pusa Purple } \\
\text { Cluster, Swaran Shamli, Pusa } \\
\text { Purple Round, Banaras Giant } \\
\text { and Arka Neelkanth }\end{array}$ & $\begin{array}{l}\text { Arka Nidhi, Pusa Purple } \\
\text { Cluster, Swaran Shamli, } \\
\text { Banaras Giant and Arka } \\
\text { Neelkanth }\end{array}$ \\
\hline $\begin{array}{l}\text { Moderately } \\
\text { resistant }\end{array}$ & $>20-40 \%$ & $\begin{array}{l}\text { Pusa Kranti, Green Long and } \\
\text { Brinjal Hybrid Gole }\end{array}$ & $\begin{array}{l}\text { Pusa Kranti and Pusa Purple } \\
\text { Round }\end{array}$ \\
\hline $\begin{array}{l}\text { Moderately } \\
\text { susceptible }\end{array}$ & $>40-60 \%$ & $\begin{array}{l}\text { Pusa Hybrid-5, Navkiran, } \\
\text { Punjab Sadabahar, Shamli, } \\
\text { Brinjal Round, Nisha Improved, } \\
\text { Pusa Hybrid- 6, Pusa Hybrid- } \\
13 \text { and Unnati }\end{array}$ & $\begin{array}{l}\text { PusaHybrid-5,Punjab } \\
\text { Sadabahar, Shamli, Nisha } \\
\text { Improved, Pusa Hybrid-6, } \\
\text { Green long, Pusa Hybrid- } 13 \\
\text { Brinjal Hybrid Gole and Unnati }\end{array}$ \\
\hline Susceptible & $>60 \%$ & $\begin{array}{l}\text { Pusa Purple Long, Kokila, } \\
\text { Manisha, Daksh, Utsav, } \\
\text { Mahadeva, Azad Kranti, } \\
\text { Krishna, Pant Samrat, Pant } \\
\text { Rituraj, Punjab Barsati and } \\
\text { Black Beauty }\end{array}$ & $\begin{array}{l}\text { Pusa Purple Long, Kokila, } \\
\text { Navkiran, Manisha, Daksh, } \\
\text { Utsav, Mahadeva, Azad Kranti, } \\
\text { Krishna, Pant Samrat, Pant } \\
\text { Rituraj, Brinjal Round, Punjab } \\
\text { Barsati and Black Beauty }\end{array}$ \\
\hline
\end{tabular}

\section{Source of Germplasm}

Germplasm
Pusa Purple Long, Pusa hybrid-5, Pusa
Hybrid-6, Pusa Hybrid 13, Pusa Kranti,
Brinjal Hybrid Gole, Navkiran
Nisha Improved, Kokila, Green Long,
Manisha, Daksh, Utsav, Mahadeva,
Unnati,
Arka Nidhi, Swaran Shamli, Pusa
Purple Round, Pusa Purple Cluster,
Punjab Sadabahar, Pant Rituraj, Pant
Samrat, Brinjal Round, Shamli, Arka
Neelkanth, Punjab Sadabahar, Black
Beauty, Uttara
Krishna, Brinjal Round,

Cultivation of resistant genotypes is the best method of managing the disease. Germplasm that were found to be resistant varieties were Pusa Purple cluster, Swaran Shamli, Arka
Source

National seed corporation Ltd., Pusa complex, New Delhi.

Century (Century seeds Pvt. Ltd.) Mangolpuri Industrial Area, New Delhi

Division of Olericulture and Floriculture, SKUAST- Jammu, Chatha, Jammu and Kashmir

Nuziveedu Seeds Limited, Connought Place, New Delhi.

Sheel in both the pot and field experiment against wilt complex. Besides resistance to soil borne pathogens, these lines could be used for hybridization programme to evolve 
cultivars possessing desirable traits. Mukherjee and Mukhopadhyay (1982) reported Pusa Purpe Long cultivar as most resistant and Pusa Kranti as moderately resistant against $R$. solanacearum. Pathania et al., (1996) reported that Arka Neelkanth and Arka Keshav were immune, whereas, Pant Rituraj, Pant Samrat and Pusa Purple Long were highly susceptible against $R$. solanacearum. Haider et al., (2001) evaluated brinjal germplasms against $M$. incognita and categorized Azad Kranti, Banaras Giant and Pant Rituraj as moderately resistance and Pusa Purple Long as susceptible cultivars.

The disease reaction (Mew and Ho, 1976):

Resistant(R): $<20 \%$ wilting

Moderately Resistant (MR): 20 - $40 \%$ wilting

Moderately Susceptible (MS): 41 - $60 \%$ wilting

Susceptible (S): > 60\% wilting

\section{Acknowledgement}

Department of Plant Pathology, Sher-eKashmir-University of Agricultural Sciences, Jammu.

\section{References}

(http://indianmedicine.eldoc.ub.rug.nl/root/Z/ $155087 /)$

(http://magazine.downtoearth.org.in/downtoea rth/15082009/) (http://vegsci.isvs.org.in/index.php/vegsci)

(http://www.chilepepperinstitute.org/content/f iles/no15.pdf)

(http://www.iospress.nl/journal/indianjournal-of-nematology/)

(http://www.worldcat.org/title/Plant\%20Disea se\%20Reporter)

Anonymous. (2009). Down to Earth: Online Science and Environment Magazine.

Anonymous. 2016. Final Estimate of 2015-16 and First Advance Estimates for 201617 of Area and Production of Horticulture Crops. Government of India, Ministry of Agriculture, Press Information Bureau.

Haider, M. G., Nath, R. P. and Srivastava, S. S. 2001. Evaluation of brinjal (Solanum melongena L.) germplasm for resistance against Meloidogyne incognita race- 2 . Indian Journal of Nematology, 31: 9394.

Mew, T. W. and Ho, W. C. 1976. Varietal resistance to bacterial wilt in tomato. Plant Disease Reporter, 60: 264-268.

Mukherjee, R. and Mukhopadhyay, S. 1982. Effect of root exudates of brinjal on Pseudomonas solanancearum. Vegetable Science, 9: 122-125.

Pathania, N. K., Singh, Y., Kalia, P. and Khar, A. 1996. Field evaluation of brinjal varieties against bacterial wilt (Pseudomonas solanacearum E. F. Smith). Capsicum and Eggplant Newsletter, 15: 67-70.

Zeven, A. C. and Zhukovsky, P. M. 1875. Dictionary of cultivated plants and their centres of diversity, pp 219. Wageningen, Netherlands.

\section{How to cite this article:}

Richa Sharma, V.K. Razdan, Stanzin Dorjey, Prerna Gupta and Seethiya Mahajan. 2018. Screening of Brinjal Germplasm against Wilt Complex Disease. Int.J.Curr.Microbiol.App.Sci. 7(08): 1534-1539. doi: https://doi.org/10.20546/ijcmas.2018.708.175 